\title{
Short OReport Asymptomatic Portal Hypertension Incidentally Detected during Upper Gastrointestinal Endoscopy
}

\section{Amit Kumar Dutta, Uday George Zachariah, Kattiparambil Gangadharan Sajith, Avinash Balekuduru, Manoj Kumar Sahu, Sudipta Dhar Chowdhury, Ashok Chacko}

\section{ABSTRACT}

Department of Gastrointestinal Sciences,

Christian Medical College,

Vellore-632 004

Tamil Nadu, India

Correspondence:

Dr. Ashok Chacko

Email: gastro@cmcvellore.ac.in
Esophageal or gastric varices may be incidentally seen during endoscopy for dyspeptic or reflux symptoms. However, the frequency of their occurrence in these patients is unknown. Our center follows the scope and treat strategy for adult patients with dyspeptic or reflux symptoms and this provided us an opportunity to study this. Apart from providing an idea on the etiological spectrum, our data suggests that patients with incidentally detected varices have well preserved liver function which may provide a window for better management.

KEYWORDS: Asymptomatic; Portal Hypertension; NAFLD

\section{Introduction}

Esophageal varices signify portal hypertension and are usually detected during endoscopy in patients with upper gastrointestinal bleed or chronic liver disease. ${ }^{1,2}$ They may also be detected incidentally during endoscopy for evaluating dyspeptic or reflux symptoms. However, the prevalence data in this setting is deficient.

\section{Methods and results}

We reviewed all the patients who had endoscopy for dyspeptic or reflux symptoms in 2008. Patients with history of jaundice, abdominal distension, lower limb swelling, dilated veins over abdominal wall, hematemesis, melena, altered sensorium, fatigue and weight loss were excluded. The total number of patients included in the study was 6227 and $21(0.34 \%)$ had varices. Among these, twenty had oesophageal and one had gastric varices. The mean age of patients with varices was $49.8+12$ years and $4(19 \%)$ were female. These patients constituted $2.9 \%$ of the total number (732) of oesophageal and/ or gastric varices detected during 2008.

All patients with varices underwent evaluation for complete blood count, liver function tests, prothrombin time, hepatitis B and $\mathrm{C}$ serology and ultrasonography of abdomen. Further evaluation was based on the physician's decision and patient compliance. All patients had well preserved liver synthetic functions (serum albumin, prothrombin time) and ultrasonography of abdomen did not show free fluid. Table 1 shows the etiology of portal hypertension in the study subjects. The commonest etiology was hepatitis B followed by non-alcoholic fatty liver disease (NAFLD). Two of the 3 patients with NAFLD were subsequently documented to have steatohepatitis on biopsy while the remaining patient had fatty 
Table 1: Etiology of incidentally detected varices

\begin{tabular}{lc}
\hline Etiology & $\begin{array}{c}\text { Number } \\
(\mathbf{n = 2 1 )}\end{array}$ \\
\hline Hepatitis B & $4(19 \%)$ \\
Non alcoholic fatty liver disease & $3(14.3 \%)$ \\
Cryptogenic chronic liver disease & $3(14.3 \%)$ \\
Non cirrhotic intrahepatic portal hypertension & $2(9.5 \%)$ \\
Hepatitis C & $1(4.8 \%)$ \\
Liver hemangioma & $1(4.8 \%)$ \\
Polycystic liver disease & $1(4.8 \%)$ \\
Alcohol & $1(4.8 \%)$ \\
Incompletely evaluated & $5(23.8 \%)$ \\
\hline
\end{tabular}

liver on ultrasonography. Two patients were diagnosed as noncirrhotic intrahepatic portal hypertension (NCIPH) after work up which included hepatic venous wedge pressure and liver biopsy. One patient had a large haemangioma $(7 \mathrm{~cm} \mathrm{x} 7 \mathrm{~cm})$ in liver while another patient had multiple cysts in the liver and kidney. In the remaining 7 patients with esophageal varices, three underwent evaluation for autoimmune liver disease; Wilson's disease, hemochromatosis and Budd-Chiari syndrome but no cause could be ascertained (cryptogenic liver disease). The other 4 patients refused further evaluation. Gastric varices were seen in one patient. The cause was not clear as ultrasonography of the abdomen with Doppler study showed normal splenic/ portal vessels and normal liver and spleen.

\section{Discussion}

This report may be the first documented series of patients where portal hypertension was not suspected prior to endoscopy. HBV infection was the commonest cause. This is not surprising as $\mathrm{HBV}$ infection is the leading cause of chronic liver disease in India. ${ }^{3,4}$ An interesting observation was the high proportion of NAFLD patients making it the second leading cause of incidentally detected portal hypertension. This and the decreasing prevalence of HBV-related disease due to increasing coverage of $\mathrm{HBV}$ vaccination, stringent guidelines about screening blood products for blood-borne viruses and improved public awareness may soon make NAFLD the leading cause of CLD and incidentally detected portal hypertension. Epidemiology and natural history of NAFLD is still being unravelled and data suggests that it affects both the obese and non-obese populations. ${ }^{5,6}$ Non-cirrhotic intrahepatic portal hypertension (NCIPH) closely mimics cryptogenic cirrhosis in its clinical presentation and laboratory profile. ${ }^{7,8}$ It is possible that NAFLD or NCIPH may well be the cause of CLD in the few patients where no cause was found and liver biopsy was not performed ${ }^{7,8}$. The absence of autoimmune liver disease and Budd-Chiari syndrome suggests that these patients are symptomatic by the time varices develop but this is conjectural. Another important observation was the well-preserved liver function in all the study subjects. In conclusion, portal hypertension incidentally detected during endoscopic evaluation of dyspepsia is not a very rare entity and merits further etiological evaluation.

\section{References}

1. Grace ND, Groszmann RJ, Garcia-Tsao G, Burroughs AK, Pagliaro L, Makuch RW, et al. Portal hypertension and variceal bleeding: an AASLD single topic symposium. Hepatology. 1998;28:868-80.

2. Mehta G, Abraldes JG, Bosch J. Developments and controversies in the management of oesophageal and gastric varices. Gut. 2010;59:701-5.

3. Batham A, Narula D, Toteja T, Sreenivas V, Puliyel JM. Sytematic review and meta-analysis of prevalence of hepatitis B in India. Indian Pediatr. 2007;44:663-74.

4. Saravanan S, Velu V, Kumarasamy N, Shankar EM, Nandakumar $\mathrm{S}$, Murugavel KG, et al. The prevalence of hepatitis B virus and hepatitis $\mathrm{C}$ virus infection among patients with chronic liver disease in South India. Int J Infect Dis. 2008;12:513-8.

5. Ruhl CE, Everhart JE. Determinants of the association of overweight with elevated serum alanine aminotransferase activity in the United States. Gastroenterology. 2003;124:71-9.

6. Das K, Das K, Mukherjee PS, Ghosh A, Ghosh S, Mridha AR, et al. Nonobese population in a developing country has a high prevalence of nonalcoholic fatty liver and significant liver disease. Hepatology. 2010;51:1593-602.

7. Maheshwari A, Thuluvath PJ. Cryptogenic cirrhosis and NAFLD: are they related? Am J Gastroenterol. 2006;101:664-8.

8. Madhu K, Avinash B, Ramakrishna B, Eapen CE, Shyamkumar $\mathrm{NK}$, Zachariah U, et al. Idiopathic non-cirrhotic intrahepatic portal hypertension: common cause of cryptogenic intrahepatic portal hypertension in a Southern Indian tertiary hospital. Indian J Gastroenterol. 2009;28:83-7. 